\title{
Editorial
}

\section{ACO: Action Coordonnée Optique}

The special issue devoted to "Action Coordonnée Optique" (ACO) opens a new topical series in EPJ Applied Physics. Thus, it is neither a conference nor a narrow scientific theme that unites these papers. Instead, this collection reflects an initiative of the French Centre National de la Recherche Scientifique (CNRS) for funding application-oriented research in the field of optics.

For the last twelve years, dramatic worldwide political and economic disruptions have deeply modified the relations between industry and academic research. The shortage of military funding, the simultaneous expansion of telecommunication technology, and the increasing pressure exerted by the global market, are among the factors that force industry and academia to renew their interactions. At our modest level we intend to echo these transformations by focusing this journal issue on the potential or effective partnership of industry and laboratories, developing under the auspices of a government institution.

We are grateful to the authors who have participated in this exploratory publication. The contributions were invited by the ACO organizers but the manuscripts were submitted to the usual peer review process.

We plan to open our journal to similar initiatives in various fields of research in the future.

Jean-Louis Le Gouët Associate Editor

\section{Introduction}

The remarkable development of optical technologies has been considerably modifying the scientific landscape in applied physics for many years already. For the past decade, progress in several fields such as: optical communication, energy resources, transportation, imaging, medical technology and bio-photonics have been stimulated by important short and long term economic objectives. In this context, beginning in 1998 the Centre National de la Recherche Scientifique (CNRS) in France decided to support multidisciplinary activities in its laboratories, aiming at encouraging the development of novel optical technologies as well as their applications to other disciplines and to the industrial world.

To achieve this goal, CNRS decided to launch a research program named Action Coordonnée Optique (ACO) in order to select and finance innovative scientific projects. A Scientific committee, composed of renowned scientists from the academic and industrial sectors, selected a variety of proposals on the basis of their general scientific quality. In addition, particular attention was given to proposals based on multidisciplinary collaborations and the ones with sound industrial perspectives. The financing of the selected projects ran for two years and the project coordinators gave oral progress reports for the Scientific Committee. The present special issue of EPJ Applied Physics is a compilation of the results from projects which were selected in 1999-2000.

The articles published in this volume constitute a mosaic of scientific topics, which makes it very interesting and unusual compared to the more traditional format found in most scientific journals. 
Therefore, the readers should not expect to find an exhaustive review of the state of the art developments in a given field. They will rather find many new trends and original ideas related to optical technologies and to a variety of their applications. We hope that the diversity and multidisciplinarity of the subjects presented here will be a source of inspiration and innovation to the readers.

The broad range of interest covered by the articles in this special issue shows the importance of optical technologies for future industrial applications. This aspect was indeed present in the ACO program which required that an industrial partner be part of each selected project. There is no doubt that we are entering a new technological era based on optical components. This new era may be compared to the preceding ones associated with the developments in electronics and computer sciences. This technological mutation is the result of growing demands of our society which include very different sectors like communications, energy resources, and health. For instance one can observe that the latest statistics show an increasing need for efficient internet access. This need, which has been constantly growing for many years, has not been perturbed by the recent, large fluctuations seen in the area of optical telecommunications. Even though these fluctuations partially affect some sectors where optics plays a role, they will not have a profound and durable impact owing to the diversity of the activities. As a matter of fact, many areas, such as: imaging, transports, and biological and medical applications, continued to develop in this less favorable climate. It should be emphasized that the current recession felt in the telecommunication sector is a characteristic of a highly nonlinear system: during the period 1995-2000, optical telecommunications developed fast to the point that the growth rate became unreasonable by 1998. This rapid increase in the development of new technologies went beyond the needs of the market, leading to a strong reaction of the investors, a process which finally stopped the development even though the actual demand from the public was constantly growing as measured by the use of internet access in the past ten years. It is therefore legitimate to think that, like for all nonlinear processes, the growth will come back very soon to a more reasonable level with less fluctuations. There is no doubt that, for the middle term, economic growth in the sectors of optical communication will start again.

In this context, the support of research agencies like the CNRS in France is extremely important in order to stimulate innovation and to prepare for the new growth. One of its important roles is to smooth economy related fluctuations. Indeed, the specific program ACO financed by CNRS continued in 2001-2002, the corresponding results being published soon. We wish to acknowledge the Director General and the Département Sciences Physiques et Mathématiques at the CNRS for their dedicated support.

The ACO piloting committee

André Ducasse

Pierre Chavel

Elisabeth Giacobino

Roland Levy 